\title{
Consumo de bebidas alcoólicas e excesso de peso em adultos brasileiros - Projeto CUME
}

\author{
Alcohol consumption and overweight in Brazilian adults \\ - CUME Project
}

Luís Paulo Souza e Souza (https://orcid.org/0000-0002-9801-4157) ${ }^{1}$

Helen Hermana Miranda Hermsdorff (https://orcid.org/0000-0002-4441-6572) ${ }^{2}$

Aline Elizabeth da Silva Miranda (https://orcid.org/0000-0001-9826-1409) ${ }^{3}$

Josefina Bressan (https://orcid.org/ 0000-0002-4993-9436) ${ }^{2}$

Adriano Marçal Pimenta (https://orcid.org/0000-0001-7049-7575) 3,4

${ }^{1}$ Departamento de

Medicina, Instituto de

Saúde e Biotecnologia,

Universidade Federal do

Amazonas. Estrada Coari/

Mamiá 305, Espírito Santo.

69.460-000 Coari AM Brasil.

${ }^{2}$ Departamento de Nutrição

e Saúde, Universidade

Federal de Viçosa. Viçosa

MG Brasil.

${ }^{3}$ Programa de Pós-

Graduação em Nutrição

e Saúde, Escola de

Enfermagem, Universidade

Federal de Minas Gerais.

Belo Horizonte MG Brasil.

${ }^{4}$ Departamento de

Enfermagem Materno-

Infantil e Saúde Pública,

Escola de Enfermagem,

Universidade Federal

de Minas Gerais. Belo

Horizonte MG Brasil.

adrianompimenta@

gmail.com
Abstract This is a cross-sectional study with 2,909 participants (aged $\geq 18$ years) from the $b a$ seline of the Cohort of Universities of Minas Gerais [CUME - Coorte de Universidades Mineiras] which verified the association between alcohol consumption and overweight. Data on sociodemographic factors, lifestyle, eating habits, anthropometric factors and clinical conditions were collected through an online questionnaire. Body mass index $\geq 25 \mathrm{~kg} / \mathrm{m}^{2}$ was considered an indicator of overweight. The daily consumption of alcohol was evaluated in grams (alcohol) and according to type in milliliters (beer, wine, hard liquor). The prevalence of alcohol consumption and overweight was $73.6 \%$ and $40.8 \%$, respectively. There was a significant tendency of an increase in overweight with higher beer consumption (tendency $p$ value of 0.038), which was not observed for the other types of alcohol. After sensitivity analyses, alcohol consumption was associated to overweight, with a tendency of increase in prevalence with higher daily consumption. There is a crucial need to curb the widely accepted idea that a low or moderate alcohol consumption is not harmful to one's health, and to be cautious of such a proposition. The influence of alcohol consumption regarding weight gain must be considered in public health policies and policies of alcohol consumption control. Key words Alcohol drinking, Overweight, Obesity
Resumo Estudo transversal com 2.909 participantes ( $\geq 18$ anos) da linha de base da Coorte de Universidades Mineiras (CUME), Brasil, que verificou a associação entre o consumo de bebidas alcoólicas e o excesso de peso. Por meio de questionário virtual, coletaram-se dados sociodemográficos, de estilo de vida, hábitos alimentares, antropométricos e condições clínicas. Considerou-se Indice de Massa Corporal $\geq 25 \mathrm{~kg} / \mathrm{m}^{2}$ como excesso de peso. Avaliou-se o consumo diário de bebidas alcoólicas no total em gramas (álcool) e segundo tipo em mililitros (cerveja, vinhos e destiladas). As prevalências de consumo de álcool e excesso de peso foram 73,6\% e 40,8\%, respectivamente. Houve uma tendência significativa de aumento da prevalência de excesso de peso quanto maior o consumo de cerveja ( $p$ de tendência $=0,038$ ), fato não observado para os demais tipos de bebidas. Após análises de sensibilidade, a ingestão de álcool associou-se ao excesso de peso, com tendência de aumento da prevalência quanto maior o consumo diário. Ressalta-se a necessidade de reduzir a visão amplamente aceita de que o consumo leve a moderado de álcool não é nocivo à saúde, adotando cautela nesta proposição. Deve-se considerar a influência da ingestão de bebidas alcoólicas no ganho de peso nas políticas públicas de saúde e de controle do consumo do álcool.

Palavras-chave Consumo de bebidas alcoólicas, Sobrepeso, Obesidade 


\section{Introdução}

O excesso de peso é a maior desordem nutricional na atualidade, sendo considerado um dos principais problemas de saúde pública. Em 2016, a prevalência mundial desse agravo em adultos foi de $39 \%^{1}$ e as despesas dos países a ele relacionadas variaram de $1 \%$ a $3 \%$ dos custos totais do setor de saúde ${ }^{2}$. No Brasil, a frequência de excesso de peso em adultos em 2018 foi de $55,7 \%{ }^{3}$, sendo que os gastos em 2017 foram de US\$2,1 bilhões ${ }^{4}$.

As causas do excesso de peso envolvem aspectos genéticos, metabólicos, psicossociais, culturais, de ingestão alimentar e sedentarismo. Em geral, os estudos que tentam explicar tais fatores têm focado principalmente no gasto energético diminuído em virtude do sedentarismo e no aumento da ingestão de alimentos altamente calóricos e das bebidas açucaradas e alcoólicas ${ }^{5-7}$.

$\mathrm{Na}$ relação entre o álcool e o excesso de peso, ao analisar as densidades calóricas das bebidas alcoólicas, nota-se se que são elevadas, pois um grama de álcool fornece 7,1 quilocalorias (kcal), sendo que para a mesma quantidade em gramas, os carboidratos fornecem $4 \mathrm{kcal}$, as proteínas 4 $\mathrm{kcal}$ e os lipídeos $9 \mathrm{kcal}^{6,8}$. Em virtude do seu valor energético, presume-se que o álcool apresente potencial para suprimir as necessidades calóricas diárias de um indivíduo e também de levá-lo ao excesso de peso, dependendo da frequência e do modo de consumo, da quantidade e do tipo de bebida $^{9-12}$.

O consumo abusivo de álcool, não considerando a dependência alcoólica, aumenta o risco para excesso de peso em ambos os sexos ${ }^{7,8,12-16}$, independentemente do tipo de bebida (vinho, cerveja e destiladas) $)^{17-20}$. Por outro lado, o consumo de bebidas alcoólicas de maneira moderada tem se mostrado um fator protetor, especialmente o vinho e a cerveja $a^{5,7,12}$.

Por apresentarem relações entre si e se constituírem como fatores de risco para as doenças e agravos não transmissíveis (DANT), que são as principais causas de morbimortalidade da população brasileira, ocasionando grande dispêndio financeiro para o Sistema Único de Saúde ${ }^{4}$, o excesso de peso e o consumo de álcool são importantes tópicos a serem investigados no campo da saúde pública ${ }^{6,21,22}$.

Apesar de a população brasileira apresentar elevado consumo médio diário de bebidas alcoólicas, poucos estudos analisaram a associação dessa exposição ao excesso de peso, acarretando incipiência e pouca consistência dos achados científicos sobre a temática no país ${ }^{23-26}$.
Este estudo também se faz relevante por avaliar uma parcela da população brasileira pouco explorada em investigações epidemiológicas, distribuída em todo o território nacional, com maior nível educacional, ocupante de cargos e funções importantes para a economia do país, com altas prevalências de hábitos nocivos à saú$\mathrm{de}^{27}$. Portanto, o adoecimento e o óbito dessa população podem provocar ônus sociais e econômicos elevados.

Dessa forma, objetivou-se verificar a associação entre o consumo de bebidas alcoólicas e o excesso de peso em adultos da linha de base da Coorte de Universidades Mineiras (CUME), Brasil.

\section{Método}

Trata-se de um estudo transversal, referente à análise da linha de base da Coorte de Universidades Mineiras (CUME), coorte aberta e de grupo populacional restrito, que visa avaliar o impacto do padrão alimentar brasileiro e da transição nutricional sobre as DANT em egressos de Instituições Federais de Ensino Superior (IFES) situadas em Minas Gerais, região Sudeste do Brasil. O delineamento, as estratégias de divulgação empregadas e o perfil da linha de base dos participantes do projeto foram detalhados em publicação prévia $^{27}$.

A coleta de dados ocorreu entre março e agosto de 2016, em ambiente virtual próprio do projeto CUME, com egressos da Universidade Federal de Viçosa (UFV) e da Universidade Federal de Minas Gerais (UFMG), que foram convidados via e-mail. Aqueles que concordaram com o termo de consentimento livre e esclarecido tiveram acesso ao instrumento de coleta de dados.

O convite para participar da pesquisa foi enviado para todos os 64.202 ex-alunos da UFMG e 16.945 ex-alunos da UFV formados entre $1994 \mathrm{e}$ 2014 (graduados e pós-graduados) cujos e-mails constavam nas bases de dados das associações de ex-alunos ou nas diretorias de tecnologia e informação das IFES. Um total de 4.949 participantes responderam ao questionário da linha de base (Q_0), representando uma taxa de resposta de 6,1\%.

Para a análise deste artigo, foram excluídos aqueles com preenchimento incompleto do Questionário de Frequência de Consumo Alimentar-QFCA ( $\mathrm{n}=1.679)$; gestantes e mulheres que tiveram filho no último ano $(\mathrm{n}=123)$; que relataram valores inconsistentes para o consumo total de calorias $[<500 \mathrm{kcal} / \mathrm{dia}(\mathrm{n}=1)$ ou 
$>6.000 \mathrm{kcal} / \mathrm{dia}(\mathrm{n}=92)]^{28}$; egressos de outras nacionalidades $(\mathrm{n}=11)$; e brasileiros residentes no exterior $(\mathrm{n}=134)$. Assim, a amostra final consistiu em 2.909 participantes.

Para coleta dos dados, utilizou-se questionário online basal, que era autoadministrado e foi dividido em duas partes devido à sua extensão (acesso em: http://www.projetocume.com.br/ questionario). A primeira parte era composta por questões relativas às características sociodemográficas, econômicas, de estilo de vida, morbidade referida individual e familiar, uso de medicamentos, histórico pessoal de exames clínicos e bioquímicos dos últimos dois anos e dados antropométricos. A segunda parte foi enviada uma semana após a conclusão da primeira e trazia o QFCA, composto por 144 itens alimentares baseados em versão original previamente validada no Brasil, referente ao consumo no último ano ${ }^{29}$.

A variável de desfecho foi o "excesso de peso", definida com base nos dados autorreferidos de peso (em quilos) e altura (em centímetros), calculando-se o Índice de Massa Corporal (IMC). As pessoas foram classificadas de duas formas: IMC $<25,0 \mathrm{~kg} / \mathrm{m}^{2}=$ sem excesso de peso; IMC $\geq$ $25,0 \mathrm{~kg} / \mathrm{m}^{2}=$ com excesso de peso.

Os dados autorreferidos de peso, altura e IMC foram previamente validados em estudo conduzido com uma subamostra da CUME, obtendo-se coeficientes de correlação intraclasse que indicavam excelente concordância: 0,989 (peso), 0,995 (altura) e 0,983 (IMC) $)^{30}$.

Para avaliação do consumo de bebidas alcoólicas, foram utilizadas informações obtidas no QFCA. Na parte destinada ao consumo de bebidas, para cada uma selecionada (cachaça; destiladas = rum, vodka e whisky; cerveja; vinhos), o participante indicou o tamanho da porção alimentar expressa em medidas caseiras comumente utilizadas no Brasil (copos, taças, latas) e a frequência usual de consumo (dia/semana/mês/ ano).

As frequências do consumo de cada bebida foram transformadas em frequências diárias que por sua vez foram multiplicadas pelo tamanho das porções para cálculos dos consumos diários das bebidas (gramas - g, ou mililitros - ml). Para os cálculos da ingestão de álcool e de cada tipo de bebida alcoólica (cerveja, vinho e destilada), utilizaram-se tabelas brasileiras de composição nutricional de alimentos ${ }^{31}$ e, quando necessária, a tabela do Departamento de Agricultura dos Estados Unidos ${ }^{32}$.

Ao final, foram consideradas como variáveis de exposição: a) consumo de bebidas alcoólicas (não, sim); b) frequência semanal do consumo de bebidas alcoólicas ( $<1$ dia/semana; 1-4 dias/ semana; $\geq 5$ dias/semana); c) consumo diário de álcool (g) (em quartis); d) consumo diário de tipos de bebidas alcoólicas (ml) (em quartis) = d1) cerveja, d2) vinho e d3) destiladas = cachaça, rum, vodka e whisky.

Os consumos diários de cada bebida alcoólica e de álcool, além das covariáveis relacionadas ao consumo alimentar, foram ajustados pela ingestão calórica, utilizando-se o método residual ${ }^{33}$, previamente às análises estatísticas.

Como covariáveis, consideraram-se: a) $c a-$ racterísticas sociodemográficas: sexo (masculino, feminino); idade (anos - contínua); cor (branca, preta/parda, amarela/indígena); estado civil (solteiro, casado legalmente/união estável, separado ou divorciado/viúvo); situação profissional (aposentado/do lar/desempregado, estudante, trabalho em tempo integral, trabalho em tempo parcial, trabalho informal) e renda familiar (contínua); b) hábitos de vida: tabagismo (nunca fumante, ex-fumante e fumante atual) e atividade física, avaliada por meio de uma lista de 24 atividades e expressa em minutos por semana (ativo = indivíduos com $\geq 150$ minutos/semana de atividade de intensidade moderada ou $\geq 75 \mathrm{mi}$ nutos/semana de atividade de intensidade vigorosa; insuficientemente ativo = indivíduos com $<$ 150 minutos/semana de atividade de intensidade moderada ou $<75$ minutos/semana de atividade de intensidade vigorosa; inativo = ausência de atividade física no lazer $)^{34}$; c) características clínicas: autorrelato de diagnóstico médico para doenças crônicas: acidente vascular encefálico, infarto, doença celíaca, doenças inflamatórias intestinas - Crohn, retocolite ulcerativa, esteatose hepática não-alcoólica, cirrose alcoólica, gastrite, úlcera gástrica ou duodenal, insuficiência renal aguda, insuficiência renal crônica, câncer de pulmão, câncer de pele, câncer de cólon, câncer de mama, câncer de colo de útero, câncer de próstata, diabetes mellitus tipo 2, hipertensão arterial sistêmica, triglicerídeos alto, colesterol alto, depressão (não, sim); d) consumo alimentar: estas variáveis também foram obtidas do QFCA, conforme metodologia semelhante à empregada para as estimativas de consumo de álcool, sendo, portanto, calculadas em gramas diárias de cada alimento. Para os cálculos das ingestões calórica (kcal) e de nutrientes também foram utilizadas tabelas brasileiras de composição nutricional dos alimentos $^{31}$ e a tabela do Departamento de Agricultura dos Estados Unidos ${ }^{32}$. Os itens alimentares foram classificados, de acordo com a classi- 
ficação NOVA, em minimamente processados, processados e ultraprocessados ${ }^{35}$.

A caracterização dos participantes foi realizada com a distribuição de frequências absolutas e relativas, médias e desvios-padrão das variáveis sociodemográficas, econômicas, de estilo de vida e de condições de saúde, segundo a presença ou não do excesso de peso. Diferenças estatísticas foram avaliadas com os testes qui-quadrado de Pearson ou t-Student.

Razões de prevalência (RP) e seus respectivos intervalos de confiança (IC) de 95\% das associações entre cada variável de exposição e o excesso de peso foram estimados por meio de modelos de regressão de Poisson com variâncias robustas, ajustando inicialmente por sexo e idade, e posteriormente por cor da pele, estado civil, situação profissional, hábito de fumar, atividade física, diagnóstico médico de depressão, renda familiar, álcool total e consumo total de energia.

Realizaram-se análises de sensibilidade para avaliar a robustez dos resultados, excluindo: (a) pessoas com diagnóstico médico prévio de obesidade, (b) pessoas que ganharam $10 \mathrm{~kg}$ ou mais previamente ao estudo, (c) todas as pessoas consideradas nas análises $\mathrm{a} \mathrm{e}$, adicionalmente àquelas que usavam medicação para controle do peso.

Os dados foram analisados no software Stata versão 13.0, com nível de significância estatística de $5 \%$. O estudo foi aprovado pelos comitês de Ética em Pesquisa com Seres Humanos da UFV e da UFMG.

\section{Resultados}

Do total de participantes, $40,8 \%(\mathrm{n}=1.188)$ estavam com excesso de peso, $27,6 \%(\mathrm{n}=804)$ estavam com sobrepeso e $13,2 \%(\mathrm{n}=384)$ eram obesos. Além disso, 3,0\% $(\mathrm{n}=87)$ estavam com baixo peso e $56,2 \%(n=1.634)$ eram eutróficos.

Comparados aos participantes sem excesso de peso, os acima do peso eram, em maior proporção, do sexo masculino, casados, com trabalho em tempo integral e com maiores médias de idade e de renda familiar $(\mathrm{p}<0,05)$ (Tabela 1$)$.

Além disso, os participantes com excesso de peso apresentavam maiores frequências de tabagismo ou ex-tabagismo, inatividade física e diagnósticos médicos de depressão e de doenças crônicas, além de terem maiores médias de ingestão calórica (kcal/dia) e de consumo de macronutrientes (g/dia) [carboidratos, proteínas, lipídeos (ácidos graxos poli-insaturados - PUFA, ácidos graxos monoinsaturados - MUFA, gordura saturada, gordura trans)] e menor média de consumo de alimentos minimamente processados $(\mathrm{p}<$ 0,05) (Tabela 2).

Em relação à ingestão de álcool, 73,6\% fizeram consumo, sendo que a média diária do consumo de álcool foi igual a 5,9 g (desvio-padrão - DP = 9,1 g). Analisando os tipos de bebidas, o consumo médio diário de cerveja foi de $76,2 \mathrm{ml}$ $(\mathrm{DP}=22,6 \mathrm{ml})$; o de vinhos foi de $16,1 \mathrm{ml}(\mathrm{DP}=$ $5,0 \mathrm{ml}$ ); e o de destiladas foi igual a $2,9 \mathrm{ml}$ (DP $=1,6 \mathrm{ml}$ ).

Os participantes com excesso de peso, comparados àqueles sem tal resultado, apresentaram maiores frequências de consumo de álcool entre um e quatro dias por semana e maiores médias de consumo diário de álcool, cerveja, vinhos e destiladas $(\mathrm{p}<0,05)$ (Tabela 3$)$.

$\mathrm{Na}$ análise multivariada, os participantes no quartil intermediário de ingestão diária de álcool (Q2 - RP: 0,87; IC: 0,77-0,99) tinham menores prevalências de excesso de peso na comparação com o menor quartil. $\mathrm{Na}$ análise por tipos de bebidas, os participantes nos quartis intermediários de consumo de cerveja (Q2 - RP: 0,84; IC 95\%: 0,74-0,96), destiladas (Q3 - RP: 0,84; IC 95\%: 0,74-0,94) e vinhos (Q3 - RP: 0,85; IC 95\%: 0,75$0,96)$ tinham menores prevalências de excesso de peso, comparados àqueles dos menores quartis. Contudo, houve tendência significativa de aumento da prevalência do excesso de peso quanto maior foi o consumo de cerveja $(\mathrm{p}$ de tendência $=$ 0,038 ), fato não observado para os demais tipos de bebidas $(\mathrm{p} \geq 0,05)$ (Tabela 4$)$.

As análises de sensibilidade mostraram que: quando se excluíram (1) os participantes com diagnóstico médico prévio de obesidade $(\mathrm{n}=$ 256), (2) os que ganharam dez ou mais quilogramas previamente ao estudo $(\mathrm{n}=203)$ ou $(3)$ a combinação dos dois critérios + participantes que faziam uso de medicamentos para perder peso $(n=420)$, passou-se (a) a verificar uma tendência de aumento da prevalência do excesso de peso quanto maior a ingestão de álcool nos critérios de exclusão 1 e 3 , e de cerveja em todos os critérios de exclusão ( $\mathrm{p}$ de tendência $<0,05$ ), e (b) perdeu-se a associação negativa entre o consumo intermediário de vinho (Q2) e o excesso de peso, e o consumo intermediário de bebidas destiladas (Q3) passou a se associar negativamente ao excesso de peso em todos os critérios de exclusão (Tabela 5). 
Tabela 1. Características sociodemográficas dos participantes de acordo com a presença do excesso de peso. Coorte de Universidades Mineiras (CUME), 2016.

\begin{tabular}{|c|c|c|c|c|c|c|c|}
\hline \multirow{3}{*}{ Variáveis } & \multicolumn{6}{|c|}{ Excesso de peso } & \multirow{3}{*}{ p-valor ${ }^{*}$} \\
\hline & \multicolumn{2}{|c|}{$\begin{array}{c}\text { Total } \\
(\mathrm{n}=2.909)\end{array}$} & \multicolumn{2}{|c|}{$\begin{array}{c}\text { Sim } \\
(\mathrm{n}=1.188)\end{array}$} & \multicolumn{2}{|c|}{$\begin{array}{c}\text { Não } \\
(\mathrm{n}=1.721)\end{array}$} & \\
\hline & n & $\%$ & $\mathbf{n}$ & $\%$ & n & $\%$ & \\
\hline Sexo & & & & & & & $<0,001$ \\
\hline Masculino & 909 & 31,3 & 525 & 44,2 & 384 & 22,3 & \\
\hline Idade $^{\star *}$ & \multicolumn{2}{|c|}{$36,3(9,5)$} & \multicolumn{2}{|c|}{$39,8(10,7)$} & \multicolumn{2}{|r|}{$33,9(7,8)$} & $<0,001$ \\
\hline Cor da pele & & & & & & & 0,202 \\
\hline Branca & 1.896 & 65,2 & 753 & 63,4 & 1.143 & 66,4 & \\
\hline Preta/parda & 984 & 33,8 & 421 & 35,4 & 563 & 32,7 & \\
\hline Amarela/indígena & 29 & 1 & 14 & 1,2 & 15 & 0,9 & \\
\hline Estado civil & & & & & & & $<0,001$ \\
\hline Solteiro & 1.276 & 43,9 & 428 & 36 & 848 & 49,3 & \\
\hline Casado legalmente/união estável & 1.465 & 50,4 & 669 & 56,3 & 796 & 46,2 & \\
\hline Separado ou divorciado/viúvo & 168 & 5,7 & 91 & 7,7 & 77 & 4,5 & \\
\hline Situação profissional & & & & & & & $<0,001$ \\
\hline Aposentado/do lar/desempregado & 235 & 8,1 & 110 & 9,2 & 125 & 7,3 & \\
\hline Estudante & 382 & 13,1 & 107 & 9 & 275 & 15,9 & \\
\hline Trabalho em tempo integral & 1.857 & 63,8 & 805 & 67,8 & 1.052 & 61,1 & \\
\hline Trabalho em tempo parcial & 358 & 12,3 & 138 & 11,6 & 220 & 12,8 & \\
\hline Trabalho informal & 77 & 2,7 & 28 & 2,4 & 49 & 2,9 & \\
\hline Renda familiar $(\mathrm{R} \$)^{* *}$ & 8.75 & $6.901)$ & 9.344 & 7.343) & & $8(6.901)$ & $<0,001$ \\
\hline
\end{tabular}

Notas: ${ }^{\star}$ valores de p segundo teste qui-quadrado de Pearson ou teste t-Student, quando apropriado; ${ }^{\star \star}$ os dados são médias e desvios-padrão.

Fonte: Elaborado pelos autores.

\section{Discussão}

Foram evidenciadas altas prevalências de consumo de bebidas alcoólicas e de excesso de peso entre os participantes. Por outro lado, as médias da ingestão diária total de álcool e dos tipos de bebidas alcoólicas foram baixas. Destaca-se, ainda, que os consumos de álcool, cerveja, vinhos e destiladas em quartis intermediários se associaram negativamente ao excesso de peso. Entretanto, após as análises de sensibilidade, tal achado se manteve apenas para as bebidas destiladas, enquanto os consumos de álcool e cerveja passaram a se associar positivamente ao desfecho.

A proporção de participantes que consumiam algum tipo de bebida alcoólica $(73,6 \%)$ foi maior do que aquelas encontradas para a população mundial $(43 \%)$ e brasileira em geral $(40 \%)^{36}$. Por outro lado, o consumo médio diário de álcool $(5,9 \mathrm{~g})$ de nossos participantes esteve abaixo do valor encontrado para a população mundial, que foi de $33 \mathrm{~g}$ (equivalente a $300 \mathrm{ml}$ de vinho; ou $750 \mathrm{ml}$ de cerveja; ou $80 \mathrm{ml}$ de destiladas $)^{36}$, e dentro dos limites de ingestão recomendados pela Organização Mundial da Saúde (10 g a 12 $\mathrm{g}$ - correspondendo a uma taça de vinho de 100 $\mathrm{ml}$, uma lata de cerveja de $330 \mathrm{ml}$ ou uma dose de bebida destilada de $30 \mathrm{ml})^{37}$. Destaca-se que as quantidades de consumo seguro e as recomendações sobre a ingestão diária máxima de álcool são diferentes entre e dentro dos países, não apresentando consenso quanto aos limites de consumo $^{37,38}$, que varia de 10 a $14 \mathrm{~g}$. Estudo publicado recentemente pelo Global Burden of Disease $(\mathrm{GBD})^{36}$ desencoraja qualquer quantidade de consumo ("tolerância zero").

$\mathrm{Na}$ análise por tipo de bebidas, houve maior consumo médio diário de cerveja $(76,2 \mathrm{ml})$, seguido dos vinhos $(16,1 \mathrm{ml})$ e das destiladas $(2,9$ $\mathrm{ml})$. Mundialmente, essas últimas correspondem ao tipo mais consumido $(44,8 \%)$, seguido da cerveja $(34,3 \%)$ e dos vinhos (11,7\%). Nas Américas, a cerveja é o tipo de bebida mais consumido (53,8\%), seguido das destiladas (31,7\%) e do vinho (13,5\%). No Brasil, o resultado é semelhante (cerveja: 62\%; destiladas: $34 \%$; vinhos: $3 \%$ ), co- 
Tabela 2. Características dos hábitos de vida, consumo alimentar e diagnóstico médico de doenças dos participantes de acordo com a presença do excesso de peso. Coorte de Universidades Mineiras (CUME), 2016.

\begin{tabular}{|c|c|c|c|c|c|c|c|}
\hline \multirow{3}{*}{ Variáveis } & \multirow{2}{*}{\multicolumn{2}{|c|}{$\begin{array}{c}\text { Total } \\
(\mathrm{n}=2.909)\end{array}$}} & \multicolumn{4}{|c|}{ Excesso de peso } & \multirow{3}{*}{ p-valor } \\
\hline & & & \multicolumn{2}{|c|}{$\begin{array}{c}\text { Sim } \\
(\mathrm{n}=1.188)\end{array}$} & \multicolumn{2}{|c|}{$\begin{array}{c}\text { Não } \\
(\mathrm{n}=1.721)\end{array}$} & \\
\hline & $\mathbf{n}$ & $\%$ & $\mathbf{n}$ & $\%$ & n & $\%$ & \\
\hline Hábito de fumar & & & & & & & $<0,001$ \\
\hline Nunca fumante & 2.303 & 79,2 & 870 & 73,2 & 1.433 & 83,3 & \\
\hline Ex-fumante & 353 & 12,1 & 197 & 16,6 & 156 & 9,1 & \\
\hline Fumante atual & 253 & 8,7 & 121 & 10,2 & 132 & 7,6 & \\
\hline Atividade física & & & & & & & 0,016 \\
\hline Inativo & 706 & 24,3 & 321 & 27 & 385 & 22,4 & \\
\hline Insuficientemente ativo & 595 & 20,4 & 236 & 19,9 & 359 & 20,8 & \\
\hline Ativo & 1.608 & 55,3 & 631 & 53,1 & 977 & 56,8 & \\
\hline Consumo de energia $(\mathrm{Kcal} / \mathrm{dia})^{* \star}$ & \multicolumn{2}{|c|}{$2.412(939)$} & \multicolumn{2}{|c|}{$2.568(991)$} & \multicolumn{2}{|c|}{$2.304(887)$} & $<0,001$ \\
\hline \multicolumn{8}{|l|}{ Consumo de macronutrientes $(\mathrm{g} / \mathrm{dia})^{* *}$} \\
\hline Carboidratos & \multicolumn{2}{|c|}{$283,4(116,3)$} & \multicolumn{2}{|c|}{$296,3(121,7)$} & \multicolumn{2}{|c|}{$274,5(111,7)$} & $<0,001$ \\
\hline Proteínas & \multicolumn{2}{|c|}{$107,8(54,9)$} & \multicolumn{2}{|c|}{$116,4(56,1)$} & \multicolumn{2}{|c|}{$101,9(52,3)$} & $<0,001$ \\
\hline Lipídeos & \multicolumn{2}{|c|}{$89,5(45,8)$} & \multicolumn{2}{|c|}{$96,5(48,7)$} & \multicolumn{2}{|c|}{$84,7(43,1)$} & $<0,001$ \\
\hline Gordura poli-insaturada & \multicolumn{2}{|c|}{$16,1(9,1)$} & \multicolumn{2}{|c|}{$17,3(9,7)$} & \multicolumn{2}{|c|}{$15,2(8,5)$} & $<0,001$ \\
\hline Gordura monoinsaturada & \multicolumn{2}{|c|}{$37,9(21,1)$} & \multicolumn{2}{|c|}{$40,9(22,4)$} & \multicolumn{2}{|c|}{$35,9(20,1)$} & $<0,001$ \\
\hline Gordura saturada & \multicolumn{2}{|c|}{$31,4(16,3)$} & \multicolumn{2}{|c|}{$34,2(17,7)$} & \multicolumn{2}{|c|}{$29,4(15)$} & $<0,001$ \\
\hline Gordura trans & \multicolumn{2}{|c|}{$1,1(0,8)$} & \multicolumn{2}{|c|}{$1,2(0,9)$} & \multicolumn{2}{|c|}{$0,9(0,7)$} & $<0,001$ \\
\hline Alimentos minimamente processados $(\mathrm{g} / \mathrm{dia})^{\star \star}$ & \multicolumn{2}{|c|}{$1.292,9(399,5)$} & $1.268,6$ & $409,1)$ & $1.309,6$ & $(391,9)$ & 0,006 \\
\hline Alimentos processados $(\mathrm{g} / \mathrm{dia})^{\star *}$ & & $(56,7)$ & & $2(56)$ & & $(57,2)$ & 0,076 \\
\hline Alimentos ultraprocessados $(\mathrm{g} / \mathrm{dia})^{\star \star *}$ & 227,8 & $(117,6)$ & 226 & $121,4)$ & & $9(115)$ & 0,494 \\
\hline Presença de doença $(\mathrm{s})$ crônica $(\mathrm{s})^{* * *}$ & & & & & & & \\
\hline Sim & 664 & 22,8 & 303 & 25,5 & 361 & 21 & 0,004 \\
\hline Diagnóstico médico de depressão & & & & & & & \\
\hline Sim & 353 & 12,1 & 181 & 15,2 & 172 & 10 & $<0,001$ \\
\hline
\end{tabular}

Notas: g: gramas; Kcal: calorias; ${ }^{\star}$ valores de p segundo teste qui-quadrado de Pearson ou teste t-Student, quando apropriado; ${ }^{\star \star}$ os dados são médias e desvios-padrão; ${ }^{* *}$ a variável doença crônica foi criada a partir da indicação pelo participante de diagnóstico médico das seguintes doenças: acidente vascular encefálico, infarto, doença celíaca, doenças inflamatórias intestinas - Crohn, retocolite ulcerativa, esteatose hepática não-alcoólica, cirrose alcoólica, gastrite, úlcera gástrica ou duodenal, insuficiência renal aguda, insuficiência renal crônica, câncer de pulmão, câncer de pele, câncer de cólon, câncer de mama, câncer de colo de útero, câncer de próstata, diabetes mellitus tipo 2, hipertensão arterial sistêmica, triglicerídeos alto, colesterol alto.

Fonte: Elaborado pelos autores.

locando o país como o terceiro na América Latina e o quinto em todo o continente com o maior consumo de álcool ${ }^{36}$.

O fato de os participantes do nosso estudo relatarem em alta proporção o consumo de bebidas alcoólicas, porém em baixa quantidade diária, pode ser considerado um aspecto comportamental positivo, tendo em vista que elevadas quantidades de álcool ingeridas por dia podem ser nocivas à saúde ${ }^{36}$. Tal resultado deve, potencialmente, estar sendo influenciado pela alta escolaridade, pois em geral indivíduos com maiores níveis educacionais tendem a apresentar hábitos de vida mais saudáveis ${ }^{39}$.
Os consumos intermediários de álcool $(\mathrm{Q} 2$ : 1,18 a 3,3g/dia), de cerveja (Q2: 8,1 a $31 \mathrm{ml} /$ dia), de vinhos (Q3: 6,4 a 17,6 ml/dia) e de bebidas destiladas (Q3: 1,1 a 2,5 ml/dia) se associaram negativamente ao excesso de peso, sugerindo uma associação inversa, apesar do $\mathrm{p}$ de tendência se apresentar significativo apenas na análise do consumo diário de cerveja, o que indica que a ingestão mais elevada dessa bebida aumenta a prevalência do desfecho. Sobre esses achados, outros estudos encontraram resultados similares ${ }^{10,14,38,40}$.

Entre os consumidores moderados e pesados de bebidas alcoólicas, uma fração maior da energia do álcool pode não ser uma fonte ener- 
Tabela 3. Características do consumo de álcool dos participantes de acordo com a presença do excesso de peso. Coorte de Universidades Mineiras (CUME), 2016.

\begin{tabular}{|c|c|c|c|c|c|c|c|}
\hline \multirow[t]{2}{*}{ Variáveis } & \multicolumn{2}{|c|}{$\begin{array}{c}\text { Total } \\
(\mathrm{n}=2.909)\end{array}$} & \multicolumn{2}{|c|}{$\begin{array}{c}\text { Sim } \\
(\mathrm{n}=1.188)\end{array}$} & \multicolumn{2}{|c|}{$\begin{array}{c}\text { Não } \\
(\mathrm{n}=1.721)\end{array}$} & \multirow[t]{2}{*}{ p-valor ${ }^{*}$} \\
\hline & $\mathbf{n}$ & $\%$ & n & $\%$ & $\mathbf{n}$ & $\%$ & \\
\hline Consumo de álcool & & & & & & & 0,010 \\
\hline Não & 767 & 26,4 & 283 & 23,8 & 484 & 28,1 & \\
\hline Sim & 2.142 & 73,6 & 905 & 76,2 & 1.237 & 71,9 & \\
\hline Frequência do consumo & & & & & & & 0,002 \\
\hline$<1 \mathrm{vez} /$ semana & 1.824 & 62,7 & 702 & 59,1 & 1.122 & 65,2 & \\
\hline 1-4 dias/semana & 1.047 & 36,0 & 466 & 39,2 & 581 & 33,8 & \\
\hline$\geq 5$ dias/semana & 38 & 1,3 & 20 & 1,7 & 18 & 1,0 & \\
\hline Consumo diário de álcool $(\mathrm{g})^{\star *}$ & \multicolumn{2}{|c|}{$5,9(9,1)$} & \multicolumn{2}{|c|}{$6,6(9,9)$} & \multicolumn{2}{|c|}{$5,4(8,4)$} & $<0,001$ \\
\hline Consumo diário de álcool & & & & & & & $<0,001$ \\
\hline Q1 (até 1,17 g) & 728 & 25,0 & 308 & 25,9 & 420 & 24,4 & \\
\hline Q2 (até 3,3 g) & 727 & 25,0 & 250 & 21,0 & 477 & 27,7 & \\
\hline Q3 (até $8 \mathrm{~g}$ ) & 727 & 25,0 & 274 & 23,1 & 453 & 26,3 & \\
\hline Q4 (até $117,3 \mathrm{~g}$ ) & 727 & 25,0 & 356 & 30,0 & 371 & 21,6 & \\
\hline Consumo diário de cerveja $(\mathrm{ml})^{\star *}$ & \multicolumn{2}{|c|}{$76,2(22,6)$} & \multicolumn{2}{|c|}{$80,0(23,9)$} & \multicolumn{2}{|c|}{$73,6(21,4)$} & $<0,001$ \\
\hline Consumo diário de cerveja & & & & & & & $<0,001$ \\
\hline Q1 (até $8 \mathrm{~mL}$ ) & 728 & 25,0 & 316 & 26,7 & 412 & 23,9 & \\
\hline Q2 (até $31 \mathrm{~mL}$ ) & 727 & 25,0 & 242 & 20,3 & 485 & 28,2 & \\
\hline Q3 (até 105,3 mL) & 727 & 25,0 & 274 & 23,0 & 453 & 26,3 & \\
\hline Q4 (até $2087,5 \mathrm{~mL}$ ) & 727 & 25,0 & 356 & 30,0 & 371 & 21,6 & \\
\hline Consumo diário de vinhos $(\mathrm{ml})^{\star *}$ & \multicolumn{2}{|c|}{$16,1(5,0)$} & \multicolumn{2}{|c|}{$16,9(5,3)$} & \multicolumn{2}{|c|}{$15,5(4,7)$} & $<0,001$ \\
\hline Consumo diário de vinhos & & & & & & & $<0,001$ \\
\hline Q1 (até $1,4 \mathrm{~mL}$ ) & 728 & 25,0 & 335 & 28,2 & 393 & 22,8 & \\
\hline Q2 (até 6,3 mL) & 727 & 25,0 & 283 & 23,8 & 444 & 25,8 & \\
\hline Q3 (até 17,6 mL) & 727 & 25,0 & 261 & 22,0 & 466 & 27,1 & \\
\hline Q4 (até 543,5 mL) & 727 & 25,0 & 309 & 26,0 & 418 & 24,3 & \\
\hline Consumo diário de destiladas $(\mathrm{ml})^{\star *}$ & \multicolumn{2}{|c|}{$2,9(1,6)$} & \multicolumn{2}{|c|}{$3,2(1,7)$} & \multicolumn{2}{|c|}{$2,7(1,5)$} & $<0,001$ \\
\hline Consumo diário de destiladas & & & & & & & $<0,001$ \\
\hline Q1 (até $0,1 \mathrm{~mL}$ ) & 728 & 25,0 & 349 & 29,4 & 379 & 22,0 & \\
\hline Q2 (até $1 \mathrm{~mL}$ ) & 727 & 25,0 & 288 & 24,2 & 439 & 25,5 & \\
\hline Q3 (até 2,5 mL) & 727 & 25,0 & 249 & 21,0 & 478 & 27,8 & \\
\hline Q4 (até 199,5 mL) & 727 & 25,0 & 302 & 25,4 & 425 & 24,7 & \\
\hline
\end{tabular}

Notas: Q1: primeiro quartil; Q2: segundo quartil; Q3: terceiro quartil; Q4: quarto quartil; g: gramas; ml: mililitros; *valores de p segundo teste qui-quadrado de Pearson ou teste t-Student, quando apropriado; ${ }^{\star \star}$ os dados são médias e desvios-padrão.

Fonte: Elaborado pelos autores.

gética disponível, devido à indução do sistema microssomal de oxidação do etanol (MEOS ${ }^{40}$. Na verdade, as calorias do álcool são menos contabilizadas no ganho de peso e mais em relação à toxicidade alcoólica, como a hepatotoxicidade ou a carcinogênes $\mathrm{e}^{38,40}$. Além disso, indivíduos com peso normal que consomem uma quantidade leve a moderada de álcool podem manter seus hábitos de beber sem ganhar peso, o que pode ter conferido esse efeito protetor nos quartis intermediários de nosso estudo ${ }^{38}$.
Destaca-se que boa parte dos estudos transversais, longitudinais e experimentais avaliados por revisões sistemáticas e metanálises mostram achados diferentes dos nossos, indicando associação positiva entre consumo de álcool e excesso de peso $^{5,10,12,14}$. O álcool tem um alto potencial para interagir com diferentes aspectos da regulação do peso corporal, assim, causas adicionais para resultados controversos podem decorrer dos diferentes fatores de confusão incluídos nas análises de dados ${ }^{38,40}$. Portanto, é preciso tentar controlar 
Tabela 4. Razão de prevalência e intervalo de confiança de $95 \%$ para a associação entre os padrões de consumo do álcool e o excesso de peso. Coorte de Universidades Mineiras (CUME), 2016.

\begin{tabular}{|c|c|c|c|c|}
\hline \multirow{2}{*}{ Variáveis } & \multicolumn{2}{|c|}{ Análise bruta } & \multicolumn{2}{|c|}{ Análise ajustada } \\
\hline & RP (IC 95\%) & p-valor & RP (IC 95\%) & p-valor \\
\hline \multicolumn{5}{|l|}{ Consumo de álcool ${ }^{*}$} \\
\hline Não $(\mathrm{n}=283)$ & 1,00 & - & 1,00 & - \\
\hline $\operatorname{Sim}(n=905)$ & $1,14(1,03-1,27)$ & 0,011 & $1,07(0,97-1,19)$ & 0,151 \\
\hline \multicolumn{5}{|l|}{ Frequência do consumo ${ }^{*}$} \\
\hline$<1 \mathrm{vez} /$ semana $(\mathrm{n}=702)$ & 1,00 & - & 1,00 & - \\
\hline $1-4$ dias/semana $(\mathrm{n}=466)$ & $1,15(1,05-1,26)$ & 0,001 & $1,07(0,97-1,17)$ & 0,132 \\
\hline$\geq 5$ dias/semana $(n=20)$ & $1,36(1,00-1,85)$ & 0,046 & $0,83(0,61-1,13)$ & 0,254 \\
\hline $\mathrm{p}$ tendência linear & & $<0,001$ & & 0,598 \\
\hline \multicolumn{5}{|l|}{ Consumo diário de álcool $(\mathrm{g})^{\star *}$} \\
\hline $\mathrm{Q} 1(\mathrm{n}=728)$ & 1,00 & - & 1,00 & - \\
\hline $\mathrm{Q} 2(\mathrm{n}=727)$ & $0,81(0,71-9,92)$ & 0,002 & $0,87(0,77-0,99)$ & 0,037 \\
\hline $\mathrm{Q} 3(\mathrm{n}=727)$ & $0,89(0,78-1,01)$ & 0,073 & $0,95(0,84-1,07)$ & 0,449 \\
\hline $\mathrm{Q} 4(\mathrm{n}=727)$ & $1,15(1,03-1,29)$ & 0,011 & $1,02(0,91-1,14)$ & 0,684 \\
\hline $\mathrm{p}$ tendência linear & & $<0,001$ & & 0,221 \\
\hline \multicolumn{5}{|c|}{ Consumo diário de cerveja $(\mathrm{mL})^{* *}$} \\
\hline $\mathrm{Q} 1(\mathrm{n}=728)$ & 1,00 & - & 1,00 & - \\
\hline $\mathrm{Q} 2(\mathrm{n}=727)$ & $0,76(0,67-0,87)$ & $<0,001$ & $0,84(0,74-0,96)$ & 0,011 \\
\hline Q3 ( $\mathrm{n}=727)$ & $0,86(0,76-0,98)$ & 0,027 & $0,92(0,82-1,04)$ & 0,203 \\
\hline $\mathrm{Q} 4(\mathrm{n}=727)$ & $1,12(1,00-1,26)$ & 0,034 & $1,04(0,93-1,16)$ & 0,416 \\
\hline $\mathrm{p}$ tendência linear & & $<0,001$ & & 0,038 \\
\hline \multicolumn{5}{|c|}{ Consumo diário de vinhos $(\mathrm{mL})^{\star *}$} \\
\hline $\mathrm{Q} 1(\mathrm{n}=728)$ & 1,00 & - & 1,00 & - \\
\hline $\mathrm{Q} 2(\mathrm{n}=727)$ & $0,84(0,75-0,95)$ & 0,006 & $0,92(0,82-1,03)$ & 0,175 \\
\hline $\mathrm{Q} 3(\mathrm{n}=727)$ & $0,78(0,68-0,88)$ & $<0,001$ & $0,85(0,75-0,96)$ & 0,010 \\
\hline $\mathrm{Q} 4(\mathrm{n}=727)$ & $0,92(0,82-1,03)$ & 0,178 & $0,91(0,81-1,01)$ & 0,097 \\
\hline $\mathrm{p}$ tendência linear & & 0,936 & & 0,280 \\
\hline \multicolumn{5}{|c|}{ Consumo diário de destiladas $(\mathrm{mL})^{\star *}$} \\
\hline $\mathrm{Q} 1(\mathrm{n}=728)$ & 1,00 & - & 1,00 & - \\
\hline $\mathrm{Q} 2(\mathrm{n}=727)$ & $0,82(0,73-0,92)$ & 0,001 & $0,91(0,81-1,02)$ & 0,134 \\
\hline Q3 (n = 727) & $0,71(0,62-0,81)$ & $<0,001$ & $0,84(0,74-0,94)$ & 0,005 \\
\hline $\mathrm{Q} 4(\mathrm{n}=727)$ & $0,86(0,77-0,97)$ & 0,014 & $0,93(0,84-1,04)$ & 0,237 \\
\hline $\mathrm{p}$ tendência linear & & 0,067 & & 0,325 \\
\hline
\end{tabular}

Notas: Q1: primeiro quartil; Q2: segundo quartil; Q3: terceiro quartil; Q4: quarto quartil; RP: Razão de Prevalência; IC: intervalo de confiança de 95\%; g: gramas; ml: mililitros; ${ }^{*}$ análises ajustadas por sexo, idade, cor, estado civil, situação profissional, hábito de fumar, atividade física, diagnóstico médico de depressão, renda familiar, álcool total, consumo total de energia; ${ }^{*}$ análises ajustadas por sexo, idade, cor, estado civil, situação profissional, hábito de fumar, atividade física, diagnóstico médico de depressão, renda familiar.

Fonte: Elaborado pelos autores.

esses vieses em estudos epidemiológicos, considerando, além dos aspectos já conhecidos e comumente utilizados (sexo, idade, atividade física, tabagismo, questões psicossociais, renda e situação profissional), aqueles relacionados ao padrão de uso e ingestão de álcool; ao peso corporal prévio; a fatores que podem influenciar previamente no ganho ou perda de peso, tais como enfermi- dades pregressas; à ingestão de medicamentos; ao consumo de cafeína; além de fatores genéticos individuais $^{38,40}$.

Alguns dos potenciais vieses citados são difíceis de ser controlados em estudos transversais, portanto, na tentativa de amenizá-los, optou-se pelas análises de sensibilidade em nosso estudo. Assim, foram excluídos os participantes com 
Tabela 5. Análises de sensibilidade das razões de prevalência e intervalos de confiança de $95 \%$ para a associação entre excesso de peso e padrões de consumo do álcool. Projeto CUME, 2016.

\begin{tabular}{|c|c|c|c|}
\hline \multirow[b]{2}{*}{ Variáveis } & \multicolumn{3}{|c|}{ Análises de sensibilidade } \\
\hline & $\begin{array}{c}\text { Análise } 1 \\
\text { RP (IC 95\%) }\end{array}$ & $\begin{array}{c}\text { Análise } 2 \\
\text { RP (IC 95\%) }\end{array}$ & $\begin{array}{c}\text { Análise } 3 \\
\text { RP (IC 95\%) }\end{array}$ \\
\hline \multicolumn{4}{|c|}{ Consumo diário de álcool $(\mathrm{g})^{*}$} \\
\hline $\mathrm{Q} 1(\mathrm{n}=728)$ & 1,00 & 1,00 & 1,00 \\
\hline $\mathrm{Q} 2(\mathrm{n}=727)$ & $0,90(0,77-1,04)$ & $0,90(0,78-1,04)$ & $0,90(0,76-1,06)$ \\
\hline Q3 $(\mathrm{n}=727)$ & $0,97(0,85-1,12)$ & $1,02(0,89-1,17)$ & $1,02(0,87-1,19)$ \\
\hline $\mathrm{Q} 4(\mathrm{n}=727)$ & $1,09(0,95-1,24)$ & $1,06(0,93-1,20)$ & $1,13(0,97-1,30)$ \\
\hline $\mathrm{p}$ tendência linear & 0,042 & 0,129 & 0,018 \\
\hline \multicolumn{4}{|c|}{ Consumo diário de Cerveja $(\mathrm{mL})^{*}$} \\
\hline $\mathrm{Q} 1(\mathrm{n}=728)$ & 1,00 & 1,00 & 1,00 \\
\hline $\mathrm{Q} 2(\mathrm{n}=727)$ & $0,88(0,76-1,02)$ & $0,89(0,77-1,03)$ & $0,92(0,78-1,08)$ \\
\hline Q3 $(\mathrm{n}=727)$ & $0,97(0,84-1,12)$ & $0,96(0,84-1,10)$ & $1,00(0,85-1,17)$ \\
\hline $\mathrm{Q} 4(\mathrm{n}=727)$ & $1,11(0,98-1,27)$ & $1,07(0,95-1,22)$ & $1,16(1,00-1,34)$ \\
\hline $\mathrm{p}$ tendência linear & 0,007 & 0,037 & 0,005 \\
\hline \multicolumn{4}{|c|}{ Consumo diário de Vinhos $(\mathrm{mL})^{*}$} \\
\hline $\mathrm{Q} 1(\mathrm{n}=728)$ & 1,00 & 1,00 & 1,00 \\
\hline $\mathrm{Q} 2(\mathrm{n}=727)$ & $0,97(0,85-1,11)$ & $0,96(0,84-1,10)$ & $1,01(0,87-1,17)$ \\
\hline Q3 $(\mathrm{n}=727)$ & $0,89(0,77-1,02)$ & $0,90(0,78-1,03)$ & $0,92(0,78-1,08)$ \\
\hline $\mathrm{Q} 4(\mathrm{n}=727)$ & $0,97(0,85-1,11)$ & $0,95(0,84-1,08)$ & $1,02(0,88-1,18)$ \\
\hline $\mathrm{p}$ tendência linear & 0,946 & 0,702 & 0,640 \\
\hline \multicolumn{4}{|c|}{ Consumo diário de Destiladas $(\mathrm{mL})^{*}$} \\
\hline $\mathrm{Q} 1(\mathrm{n}=728)$ & 1,00 & 1,00 & 1,00 \\
\hline $\mathrm{Q} 2(\mathrm{n}=727)$ & $0,92(0,81-1,05)$ & $0,94(0,82-1,07)$ & $0,96(0,83-1,11)$ \\
\hline Q3 $(\mathrm{n}=727)$ & $0,84(0,73-0,96)$ & $0,85(0,74-0,98)$ & $0,83(0,71-0,98)$ \\
\hline $\mathrm{Q} 4(\mathrm{n}=727)$ & $0,95(0,84-1,08)$ & $0,97(0,86-1,09)$ & $0,99(0,86-1,14)$ \\
\hline $\mathrm{p}$ tendência linear & 0,621 & 0,771 & 0,948 \\
\hline
\end{tabular}

Notas: Q1: primeiro quartil; Q2: segundo quartil; Q3: terceiro quartil; Q4: quarto quartil; RP: razão de prevalência; IC: intervalo de confiança de 95\%; g: gramas; ml: mililitros. Análise 1: exclusão das pessoas com diagnóstico médico prévio de obesidade $(\mathrm{n}=256)$. Análise 2: exclusão das pessoas que ganharam dez ou mais quilogramas previamente ao estudo $(n=203)$. Análise 3: exclusão de todas as pessoas consideradas nas análises 1 e $2(\mathrm{n}=408)$, adicionalmente àquelas que usavam medicação para controle do peso $(\mathrm{n}$ $=12)($ total $=420)$.

*Análises ajustadas por sexo, idade, cor, estado civil, situação profissional, hábito de fumar, atividade física, diagnóstico médico de depressão, renda familiar.

Fonte: Elaborado pelos autores.

diagnóstico médico prévio de obesidade, os que ganharam dez ou mais quilogramas previamente ao estudo e os que usavam medicação para controle do peso. Após esses procedimentos, apenas o consumo intermediário de bebidas destiladas (Q3: 1,1 a $2,5 \mathrm{ml} / \mathrm{dia}$ ) manteve-se relacionado negativamente ao desfecho.

Com relação aos vinhos e à cerveja, após análise de sensibilidade, nossos achados (cerveja: 8,1 a $31 \mathrm{ml} / \mathrm{dia}$; vinhos: 6,4 a $17,6 \mathrm{ml} / \mathrm{dia}$ ) diferiram daqueles encontrados por outros estudos, os quais mostraram que o consumo moderado de cerveja (11 a $22 \mathrm{ml} / \mathrm{dia})^{41}$ e de vinho (5 a 13 $\mathrm{ml} / \mathrm{dia})^{5,12}$ conferiam proteção contra o ganho de peso. Entre as possíveis elucidações, tal efeito pode ser explicado pela presença dos polifenóis dos vinhos e das cervejas, conferindo ação antioxidante e anti-inflamatória, com redução de moléculas de adesão leucocitária e biomarcadores inflamatórios, melhorando o perfil lipídico ${ }^{42}$, sendo portanto uma característica adicional favorável a essas bebidas.

Ressalta-se que essa divergência entre nossos resultados e aqueles de outras investigações pode ser explicada pelo fato de não termos separado na análise os vinhos entre tinto e branco, pois o primeiro apresenta melhores efeitos protetores na saúde devido a presença de, em média, dez vezes mais compostos fenólicos ${ }^{17,42}$. Sobre as cervejas, não foram considerados em nosso questio- 
nário os tipos separadamente. Estudo conduzido no Brasil analisou os compostos fenólicos nos diferentes tipos e estilos de cervejas nacionais, encontrando que os atributos físico-químicos (densidade, índice de refração, amargor e teor de etanol) influenciam na quantidade de fenó$\operatorname{licos}^{43}$. Além disso, o mesmo estudo identificou que o perfil fenólico das cervejas brasileiras foi distinto daquele característico das cervejas europeias, com baixos teores de ácido ferúlico ${ }^{43}$, o que poderia ser uma hipótese para conferir menor proteção em uso moderado quando comparadas às produzidas na Europa ${ }^{5,10,12,14}$. Contudo, destaca-se que, em nossas análises de tendência, verificou-se que quanto mais se aumentou o consumo de cerveja, maior foi a prevalência do excesso de peso, corroborando os resultados de outras pesquisas ${ }^{12,14,15}$.

Com relação às bebidas destiladas, estas apresentam concentrações mais elevadas de álcool e mais baixas de polifenóis, o que poderia conferir maior risco de excesso de peso ${ }^{43}$. Entretanto observou-se no presente estudo um efeito protetor no quartil intermediário (Q3: 1,1 a 2,5 ml/dia), também indo ao encontro de outros estudos ${ }^{10,12}$. Tal achado pode ser explicado pelo fato de o álcool se relacionar positivamente com o HDL- $\mathrm{c}^{42}$. Em uma metanálise de estudos prospectivos, apenas a ingestão muito leve de álcool (entre 0,1 a $5 \mathrm{~g} / \mathrm{dia}$ ) mostrou-se como fator protetor para a síndrome metabólica ${ }^{44}$. Os consumidores no quartil intermediário (Q3) de bebidas destiladas no presente estudo tinham uma mediana de consumo de álcool de 3,2 g/dia.

O Seguimiento Universidad de Navarra (SUN), na Espanha, encontrou que os bebedores de pelo menos sete drinques por semana de cerveja e destiladas apresentaram maior risco de sobrepeso e obesidade (OR: 1,32 - IC 95\%: 1,00$1,74)$ em comparação aos não-bebedores, após o ajuste multivariado dos dados ${ }^{45}$. Vale destacar que esta foi uma análise longitudinal, o que garante a causalidade das associações, fato que fortalece nossos achados.

$\mathrm{Na}$ presente investigação, observou-se ainda uma tendência de aumento da prevalência do excesso de peso quanto maior o consumo diário de álcool, o que também foi encontrado por outras pesquisas ${ }^{1,9,10,14,16,18,46}$, a despeito de outros estudos que evidenciaram associações nulas ou inversas $^{10,38,40}$.

Parte dos estudos aponta curva em forma de J nas análises do consumo de álcool e os efeitos na saúde ${ }^{1,9,10,14,16,18,46}$. Para alguns autores, esse padrão de curva em J deve ser avaliado com cuida- do, destacando que por vezes tal relação pode se dever a uma classificação errônea dos padrões de consumo. O menor risco entre os abstêmios e os que fazem uso moderado pode ser devido à inclusão de indivíduos que já apresentavam alterações e que foram orientados a diminuir o uso ou a se tornarem abstêmios ${ }^{46}$. Ressalta-se que nossas análises foram ajustadas pelo diagnóstico de doenças crônicas que potencialmente poderiam afetar o consumo de bebidas alcoólicas. Recentemente, o GBD ${ }^{36}$, apesar de encontrar a curva em J na relação entre o consumo de álcool e, sobretudo, as doenças isquêmicas, mostra que quando se avalia a mortalidade geral, a curva perde esse formato, já que o risco de morte por outras causas supera a proteção nos níveis mais baixos ou intermediários de uso. Como recomendação, o GBD enfatiza que, independentemente da quantidade, o consumo de álcool leva a alguma perda de saúde entre as populações ${ }^{36}$.

Do total de participantes, $40,8 \%$ estavam com excesso de peso. Ainda que esta prevalência seja alta, é inferior à encontrada na população adulta brasileira em geral $(55,7 \%)^{3} \mathrm{e}$ em outras pesquisas nacionais e internacionais ${ }^{28,47-50}$, mas semelhante à da coorte SUN $(38 \%)^{45}$, que apresenta amostra com características muito parecidas à nossa.

Estas diferenças podem ser explicadas pelas características da população aqui analisada, composta, majoritariamente, por adultos jovens com altas renda e escolaridade, fatores protetores para o excesso de peso'. Pessoas em melhor posição socioeconômica são mais propensas a aderirem melhor às medidas de cuidado à saúde e terem maior acesso a serviços de saúde ${ }^{39}$, ainda que possam vir a apresentar maior consumo de bebidas alcoólicas ${ }^{15,25,27,51}$.

Apesar de este estudo apresentar um perfil de amostra diferenciado, em melhor posição socioeconômica, tal característica permitiu um detalhamento e aprofundamento nas perguntas do questionário. Pesquisas com amostras semelhantes têm exibido resultados muito confiáveis e válidos, além de elevada taxa de retençã $0^{45,52}$.

Como limitação do presente estudo, cita-se o fato de ser uma análise transversal, não sendo plausível concluir relações causais. Além disso, destacamos a questão da autodeclaração do consumo de bebidas alcoólicas como possível limitador, mesmo que autores acreditem que em amostras de comunidade, em que o consumo de álcool é legalmente produzido, socialmente aceito e percebido como parte do padrão dietético tradicional, o problema da declaração incorre- 
ta pode ser minimizado ${ }^{53}$. Na coorte espanhola SUN, evidenciou-se que pessoas com maior escolaridade são menos propensas a informar falsamente o consumo de álcool ${ }^{52,54}$. Entretanto, reconhecemos que essas questões não eliminam possíveis erros de mensuração inerentes ao instrumento utilizado e às características específicas da nossa população.

Apesar de alguns estudos realizados com adultos e que utilizaram QFCA mostrarem boa reprodutibilidade e validade na aferição do consumo de "bebidas alcoólicas" $52,55,56$, além da coorte espanhola SUN ${ }^{52}$ indicar que, mesmo que haja algum erro nessa informação, é provável que ele não seja diferencial devido ao maior status educacional dos participantes, vale destacar que a estimativa de ingestão de álcool em nosso estudo pode ser também uma limitação, pois o QFCA não foi validado especificamente para esse item alimentar.

Outra limitação deste estudo foi a quantidade de perda de participantes em virtude do não preenchimento do QFCA ( $n=1.679)$. Tal situação poderia aumentar a probabilidade do erro estatístico tipo II, entretanto, mesmo após as análises multivariadas, foram evidenciadas associações significativas entre o consumo de bebidas alcoólicas e o excesso de peso. Além disso, ao se comparar os participantes com as perdas em relação a sexo, idade, frequência de consumo de bebidas alcoólicas e excesso de peso, houve apenas uma diferença estatisticamente significativa. Entre as perdas, a proporção de indivíduos do sexo masculino foi maior (39\% versus 31,3\%; p $<0,001$ pelo teste de qui-quadrado de Pearson), portanto esse achado deve ser considerado na interpretação dos nossos resultados, uma vez que homens consomem mais bebidas alcoólicas e apresentam maior prevalência de excesso de peso do que mulheres no Brasil ${ }^{3}$.
Como pontos fortes do presente estudo, destacamos que ainda são poucos os dados que efetivamente caracterizam o padrão alimentar da população brasileira, de modo que o projeto CUME poderá contribuir nesse sentido. Ao ilustrar o tamanho da porção das bebidas alcoólicas, esta pesquisa apresenta a vantagem de obter maior acurácia, o que pode minimizar o viés de memória e a subestimação esperada do consumo ${ }^{55}$. Por fim, com as análises de sensibilidade, tentou-se amenizar a possibilidade de viés de causalidade reversa, muito susceptível em estudos transversais, conforme já discutido por outros estudos que recomendam esse cuidado nas análises ${ }^{38,40}$.

Conclui-se que foi alta a prevalência do consumo de bebidas alcoólicas entre os participantes da CUME, sendo observada associação inversa nos quartis intermediários de ingestão de álcool, cerveja, vinhos e bebidas destiladas com o excesso de peso. Por outro lado, após as análises de sensibilidade, o alto consumo de álcool e de cerveja se associou positivamente ao excesso de peso, ainda que a ingestão intermediária de bebidas destiladas tenha se associado negativamente ao desfecho. Entretanto, sobre o último achado, ressalta-se a necessidade de reduzir a visão amplamente aceita de que o consumo leve a moderado de álcool não é nocivo à saúde ${ }^{5,7,12}$, no sentido de adotar cautela em tal proposição. Assim, as pessoas que fazem uso leve ou não consomem não podem ser encorajadas a esperar benefício substancial da ingestão de bebidas alcoólicas. Em contrapartida, para os que já consomem, recomenda-se moderação, evitando episódios de consumo pesado (binge drinking).

Análises longitudinais adicionais (tendo em conta a sequência temporal) são necessárias para apoiar essas associações, particularmente em populações com elevada variabilidade do consumo de álcool e avaliando o papel específico de diferentes tipos de bebidas alcoólicas.

\section{Colaboradores}

Souza e Souza LP e Miranda AES participaram da coleta, análise e interpretação dos dados. Hermsdorff HHM, Bressan J e Pimenta AM participaram da concepção e do planejamento do estudo. 


\section{Agradecimentos}

Aos participantes da Coorte de Universidades Mineiras (CUME), bem como à Coordenação de Aperfeiçoamento de Pessoal de Nível Superior (CAPES) e à Fundação de Amparo à Pesquisa do Estado de Minas Gerais (FAPEMIG) pelo apoio financeiro.

\section{Referências}

1. World Health Organization (WHO). Obesity and overweigh. Geneva: WHO; 2018.

2. Organisation for Economic Co-operation and Development (OECD) [serial on the Internet]. Obesity update; 2014 [cited 2018 Sep 01]. Available from: http:// www.oecd.org/health/Obesity-Update-2014.pdf

3. Brasil. Ministério da Saúde (MS). Vigitel Brasil 2018: vigilância de fatores de risco e proteção para doenças crônicas por inquérito telefônico: estimativas sobre frequência e distribuição sociodemográfica de fatores de risco e proteção para doenças crônicas nas capitais dos 26 estados brasileiros e no Distrito Federal em 2018. Brasília: MS; 2019.

4. Bahia L, Coutinho ES, Barufaldi LA, Abreu GA, Malhão TA, Souza CP, Araujo DV. The costs of overweight and obesity-related diseases in the Brazilian public health system: cross-sectional study. BMC Public Health 2012; 12:440-447.

5. Poppitt SD. Beverage consumption: are alcoholic and sugary drinks tipping the balance towards overweight and obesity? Nutrients 2015; 7(8):6700-6718.

6. Fazzino TL, Fleming K, Sher KJ, Sullivan DK, Befort C. Heavy Drinking in Young Adulthood Increases Risk of Transitioning to Obesity. Am J Prev Med 2017; 53(2):169-175.

7. Bezerra IN, Alencar ES. Association between excess weight and beverage portion size consumed in Brazil. Rev Saude Publica 2018; 52:21.

8. Reis NT, Rodrigues CSC. Nutrição clínica no alcoolismo. Rio de Janeiro: Rubio; 2003.

9. Kachani AT, Brasiliano S, Hochgraf PB. O impacto do consumo alcoólico no ganho de peso. Rev Psiquiatr Clín 2008; 35(supl. 1):21-24.

10. Orea CS, González MAM, Rastrollo MB. Alcohol consumption and body weight: a systematic review. Nutr Rev 2011; 69(8):419-431.

11. Brandhagen M, Forslund HB, Lissner L, Winkvist A, Lindroos AK, Carlsson LM, Sjöström L, Larsson I. Alcohol and macronutrient intake patterns are related to general and central adiposity. Eur J Clin Nutr 2012; 66(3):305-313.

12. Traversy G, Chaput JP. Alcohol consumption and obesity: an update. Curr Obes Rep 2015; 4(1):122-130.

13. Farinelli MA, Partridge SR, Roy R. Weight-related dietary behaviors in young adults. Curr Obes Rep 2016; 5:23-29.

14. Yeomans MR. Alcohol, appetite and energy balance: is alcohol intake a risk factor for obesity? Physiol Behav 2010; 100(1):82-89.

15. Costanzo S, Castelnuovo AD, Donati MB, Iacoviello L, Gaetano G. Wine, beer or spirit drinking in relation to fatal and non-fatal cardiovascular events: a meta-analysis. Eur J Epidemiol 2011; 26:833-850.

16. Bendsen NT, Christensen R, Bartels EM, Kok FJ, Sierksma A, Raben A, Astrup A. Is beer consumption related to measures of abdominal and general obesity? A systematic review and meta-analysis. Nutr Rev 2013; 71(2):67-87.

17. Lopez MTB, Rastrollo MB, Orea CS, Lopez MS, Montero AF, Gea A, González MAM. Different types of alcoholic beverages and incidence of metabolic syndrome and its components in a Mediterranean cohort. Clin Nutr 2013; 32(5):797-804. 
18. Artero A, Artero A, Tarín JJ, Cano A. The impact of moderate wine consumption on health. Maturitas 2015; 80(1):3-13.

19. Chakraborty S. Analysis of NHANES 1999-2002 data reveals noteworthy association of alcohol consumption with obesity. Ann Gastroenterol 2014; 27(3):250257.

20. Bernal RTI, Malta DC, Iser BPM, Monteiro RA. Método de projeção de indicadores das metas do Plano de Ações Estratégicas para o Enfrentamento das Doenças Crônicas não Transmissíveis no Brasil segundo capitais dos estados e Distrito Federal. Epidemiol Serv Saude 2016; 25(3):455-466.

21. Sabóia RF, Araújo AP, Barbosa JMA, Galvão CEP, Cruvel JMS, Ferreira SCN. Obesidade abdominal e fatores associados em adultos atendidos em uma clínica escola. Rev Bras Promoc Saude 2016; 29(2):259-267.

22. Cruz MF, Ramires VV, Wendt A, Mielke GI, Mesa JM, Wehrmeister FC. Simultaneity of risk factors for chronic non-communicable diseases in the elderly in Pelotas, Rio Grande do Sul State, Brazil. Cad Saude Publica 2017; 33(2):e00021916.

23. Domingos JBC, Jora NP, Carvalho AMP, Pillon SC. Consumo de álcool, sobrepeso e obesidade entre caminhoneiros. Rev Enferm UERJ 2010; 18(3):377-382.

24. Silva ABJ, Oliveira AVK, Silva JD, Quintaes KD, Fonseca VAS, Nemer ASA. Relação entre consumo de bebidas alcoólicas por universitárias e adiposidade corporal. J Bras Psiquiatr 2011; 60(3):210-215.

25. Cibeira GH, Muller C, Lazzaretti R, Nader GA, Caleffi M. Consumo de bebida alcoólica, fatores socioeconômicos e excesso de peso: um estudo transversal no sul do Brasil. Cien Saude Colet 2013; 18(12):3577-3584.

26. Carvalho CA, Fonseca PCA, Barbosa JB, Machado SP, Santos AM, Silva AAM. Associação entre fatores de risco cardiovascular e indicadores antropométricos de obesidade em universitários de São Luís, Maranhão, Brasil. Cien Saude Colet 2015; 20(2):479-490.

27. Domingos ALG, Miranda AES, Pimenta AM, Hermsdorff HHM, Oliveira FLP, Santos LC, Lopes ACS, González MAM, Bressan J. Cohort profile: The Cohort of Universities of Minas Gerais (CUME). Int J Epidemiol 2018; 47(6):1-10.

28. Schmidt MI, Duncan BB, Mill JG, Lotufo PA, Chor D, Barreto SM, Aquino EM, Passos VM, Matos SM, Molina MC, Carvalho MS, Bensenor IM. Cohort profile: Longitudinal Study of Adult Health (ELSA-Brasil). Int J Epidemiol 2015; 44(1):68-75.

29. Henn RL, Fuchs SC, Moreira LB, Fuchs FD. Development and validation of a food frequency questionnaire (FFQ-Porto Alegre) for adolescent, adult and elderly populations from Southern Brazil. Cad Saude Publica 2010; 26(11):2068-2079.

30. Miranda AES, Ferreira AVM, Oliveira FLP, Hermsdorff HHM, Bressan J, Pimenta AM. Validação da síndrome metabólica e de seus componentes autodeclarados no estudo CUME. Rev Min Enferm 2017; 21:e1069.

31. Núcleo de Estudos e Pesquisas em Alimentação. Tabela Brasileira de Composição de Alimentos. Campinas: Universidade Estadual de Campinas; 2011.
32. United States Department of Agriculture (USDA) [serial on the Internet]. National Nutrient Database for Standard Reference Release. USDA Food Composition Databases; 2018 [cited 2019 Mar 20]. Available from: https://ndb.nal.usda.gov/ndb/foods

33. Willett WC, Colditz GA. Approaches for conducting large cohort studies. Epidemiol Rev 1998; 20(1):91-99.

34. World Health Organization (WHO). Global recommendations on physical activity for health. Geneva: WHO; 2010.

35. Monteiro CA, Cannon G, Moubarac JC, Levy RB, Louzada MLC, Jaime PC. The UN Decade of $\mathrm{Nu}-$ trition, the NOVA food classification and the trouble with ultra-processing. Public Health Nutr 2018; 21(1):5-17.

36. Global Burden of Disease. Alcohol use and burden for 195 countries and territories, 1990-2016: a systematic analysis for the Global Burden of Disease Study 2016. Lancet 2018; 392(22):1015-1035.

37. Kalinowski A, Humphreys K. Governmental standard drink definitions and low-risk alcohol consumption guidelines in 37 countries. Addiction 2016; 111(7):1293-1298.

38. Wang L, Lee MI, Manson JAE, Buring JE, Sesso HD. Alcohol consumption, weight gain, and risk of becoming overweight in middle-aged and older women. Arch Intern Med 2010; 170(5):453-461.

39. Faleiro JS, Giatti L, Barreto SM, Camelo LV, Griep RH, Guimarães JMN, Fonseca MJM, Chor D, Chagas MCA. Lifetime socioeconomic status and health-related risk behaviors: the ELSA-Brazil study. Cad Saude Publica 2017; 33(3):e00017916.

40. Suter PM. Is alcohol consumption a risk factor for weight gain and obesity? Crit Rev Clin Lab Sci 2005; 42(3):197-227.

41. Romeo J, Gross MG, Wärnberg J, Díaz LE, Marcos A. Does beer have an impact on weight gain? Effects of moderate beer consumption on body composition. Nutr Hosp 2007; 22(2):223-228.

42. Huang J, Wang X, Zhang Y. Specific types of alcoholic beverage consumption and risk of type 2 diabetes: A systematic review and meta-analysis. J Diabetes Investig 2017; 8(1):56-68.

43. Nunes NM, Brito TC, Fonseca ND, Aguiar PF, Monteiro M, Perrone D, Torres AG. Phenolic compounds of Brazilian beers from different types and styles and application of chemometrics for modeling antioxidant capacity. Food Chem 2016; 15(199):105-113.

44. Sun K, Ren M, Liu D, Wang C, Yang C, Yan L. Alcohol consumption and risk of metabolic syndrome: a meta-analysis of prospective studies. Clin Nutr 2014; 33(4):596-602.

45. Orea CS, Rastrollo MB, Cordoba JMN, Gortari FJB, Beunza JJ, González MAM. Type of alcoholic beverage and incidence of overweight/obesity in a Mediterranean cohort: the SUN project. Nutrition 2011; 27(78):802-808.

46. Gaetano G, Costanzo S. Alcohol and Health. Praise of the J Curves. JACC 2017; 70(8):923-925.

47. Garcia LP, Freitas LRS. Consumo abusivo de álcool no Brasil: resultados da Pesquisa Nacional de Saúde 2013. Epidemiol Serv Saude 2015; 24(2):227-237. 
48. O’Donovan G, Stamatakis E, Hamer M. Associations between alcohol and obesity in more than 100,000 adults in England and Scotland. Br J Nutr 2018; 119(2):222-227.

49. Rezende FAC, Rosado LEPL, Ribeiro RCL, Vidigal FC, Vasques ACJ, Bonard IS, Carvalho CR. Body mass index and waist circumference: association with cardiovascular risk factors. Arq Bras Cardiol 2006; 87(6):728-734.

50. Lima NP, Horta BL, Motta JVDS, Valença MS, Oliveira V, Santos TV, Gigante DP, Barros FC. Evolution of overweight and obesity into adulthood, Pelotas, Rio Grande do Sul State, Brazil, 1982-2012. Cad Saude Publica 2015; 31(9):2017-2025.

51. Pham CV, Tran HTD, Tran NT. Alcohol consumption and binge drinking among adult population: evidence from the CHILILAB Health and Demographic Surveillance System in Vietnam. J Public Health Manag Pract 2018; 24(supl. 2):s67-sS73.

52. Gea A, Rastrollo MB, Toledo E, Lopez MG, Beunza JJ, Estruch R, González MAM. Mediterranean alcoholdrinking pattern and mortality in the SUN (Seguimiento Universidad de Navarra) Project: a prospective cohort study. Br J Nutr 2014; 28;111(10):1871-1880.

53. Lourenço S, Oliveira A, Lopes C. The effect of current and lifetime alcohol consumption on overall and central obesity. Eur J Clin Nutr 2012; 66(7):813-818.

54. Gómez MS, Fuente C, Vázquez Z, Irala J, González MAM. Cohort profile: the "Seguimiento Universidad de Navarra" (SUN) study. Int J Epidemiol 2006; 35(6):1417-1422.

55. Lopes ACS, Caiaffa WT, Mingoti SA, Costa MFFL. Food intake in epidemiological studies. Rev Bras Epidemiol 2003; 6(3):209-219.

56. Nakahata NT, Takada AN, Imaeda N, Goto C, Kuwabara KH, Niimura H, Arai Y, Yoshita K, Takezaki T. Validity of a food frequency questionnaire in a population with high alcohol consumption in Japan. Asia Pac J Clin Nutr 2016; 25(1):195-201.

Artigo apresentado em 04/04/2019

Aprovado em 25/10/2019

Versão final apresentada em 27/10/2019

Editores-chefes: Romeu Gomes, Antônio Augusto Moura da Silva 